\title{
Coronal magnetic field measurement using loop oscillations observed by Hinode/EIS
}

\author{
T. Van Doorsselaere ${ }^{1}$, V. M. Nakariakov ${ }^{1}$, P. R. Young ${ }^{2}$, and E. Verwichte ${ }^{1}$ \\ ${ }^{1}$ Centre for Fusion, Space and Astrophysics, Physics Department, University of Warwick, Coventry CV4 7AL, UK \\ e-mail: [t.van-doorsselaere;v.nakariakov; erwin.verwichte]@warwick.ac.uk \\ 2 STFC, Rutherford Appleton Laboratory, Chilton, Didcot, Oxfordshire OX11 0QX, UK \\ e-mail: p.r.young@rl.ac.uk
}

Received 13 May 2008 / Accepted 12 June 2008

\begin{abstract}
We report the first spectroscopic detection of a kink MHD oscillation of a solar coronal structure by the Extreme-Ultraviolet Imaging Spectrometer (EIS) on the Japanese Hinode satellite. The detected oscillation has an amplitude of $1 \mathrm{~km} \mathrm{~s}^{-1}$ in the Doppler shift of the FeXII $195 \AA$ spectral line (1.3 MK), and a period of $296 \mathrm{~s}$. The unique combination of EIS's spectroscopic and imaging abilities enables us to measure simultaneously the mass density and length of the oscillating loop. This enables us to measure directly the magnitude of the local magnetic field, the fundamental coronal plasma parameter, as $39 \pm 8 \mathrm{G}$, with unprecedented accuracy. This proof of concept makes EIS an exclusive instrument for the full scale implementation of the MHD coronal seismological technique.
\end{abstract}

Key words. instrumentation: spectrographs - Sun: corona - Sun: oscillations - Sun: magnetic fields

\section{Introduction}

Coronal seismology utilises observed waves and oscillations in the corona to estimate the physical properties of the coronal plasma, such as the magnetic field, which are difficult to measure directly. Alternative techniques for the measurement of the coronal magnetic field are based on infrared, radio or microwave observations, or magnetostatic extrapolation. Unfortunately, these techniques can be applied only in limited ways, such as to superstrong magnetic fields over sunspots, or have extremely low time and spatial resolution and ambiguities (see Nakariakov \& Ofman 2001, and references therein). On the other hand, the coronal seismological techniques used to estimate coronal magnetic fields have the following advantages and features: (a) the precise relation between the measurement of the magnetic field and a specific coronal structure, and as a consequence high spatial resolution (of the order of $1^{\prime \prime}$ with the current instruments); (b) both on-the-disk and off-limb measurements are possible; and (c) determination of the absolute value of the coronal magnetic field strength including all three components, which makes this technique relatively independent of LOS-effects.

The first attempts to apply this method used transverse magneto-acoustic kink oscillations (Aschwanden et al. 1999; Nakariakov et al. 1999) to measure the damping mechanism (Ofman \& Aschwanden 2002), magnetic fields (Nakariakov \& Ofman 2001), Alfvén speeds (Arregui et al. 2007), density scale heights (Andries et al. 2005a; Van Doorsselaere et al. 2007), and transverse structuring (Verwichte et al. 2006). However, these attempts suffered from the lack of a dedicated observational instrument.

The seismological measurement of the coronal magnetic field is based in general on the determination of the observed wave phase speed and the mass density of the plasma in the oscillating structure. In the case of standing waves, the phase speed can be estimated by the period of the observed oscillations and the wavelength given by the size of the resonator. The practical formula for determining the absolute value of the magnetic field $B$ in a fundamental kink oscillating loop (filled with a low- $\beta$ plasma) is

$$
B=\frac{L}{P} \sqrt{2 \mu\left(\rho_{\mathrm{in}}+\rho_{\mathrm{ex}}\right)},
$$

where $P$ is the period of oscillations, $L$ is the length of the loop, $\mu$ is the magnetic permeability, and $\rho_{\text {ex }}$ and $\rho_{\text {in }}$ are the density of the plasma outside and inside the loop, respectively (Nakariakov \& Ofman 2001). This approach was applied to estimate the field strength using the EUV imager TRACE (e.g. Nakariakov \& Ofman 2001; Verwichte et al. 2004; Van Doorsselaere et al. 2007); its applicability was however limited by the large, up to $80 \%$, errors associated with the estimate of the density and by the relative rareness of the kink oscillation phenomena, observed close to the instrumental threshold of resolution. Reliable simultaneous measurements of the observables necessary to do coronal seismology by the same instrument have not been possible because of instrumental limitations.

We present the first observations of coronal loop kink oscillations from the EIS (Extreme ultra-violet Imaging Spectrometer) instrument onboard Hinode. EIS is unique because it measures both spectroscopic and imaging information simultaneously. The presence of multiple spectral line measurements enables the emitting plasma density to be determined precisely. On the other hand, the imaging capabilities of EIS provide us with information about the waveguide. This will allow us to improve the accuracy of the magnetic field measurement using coronal seismology. 

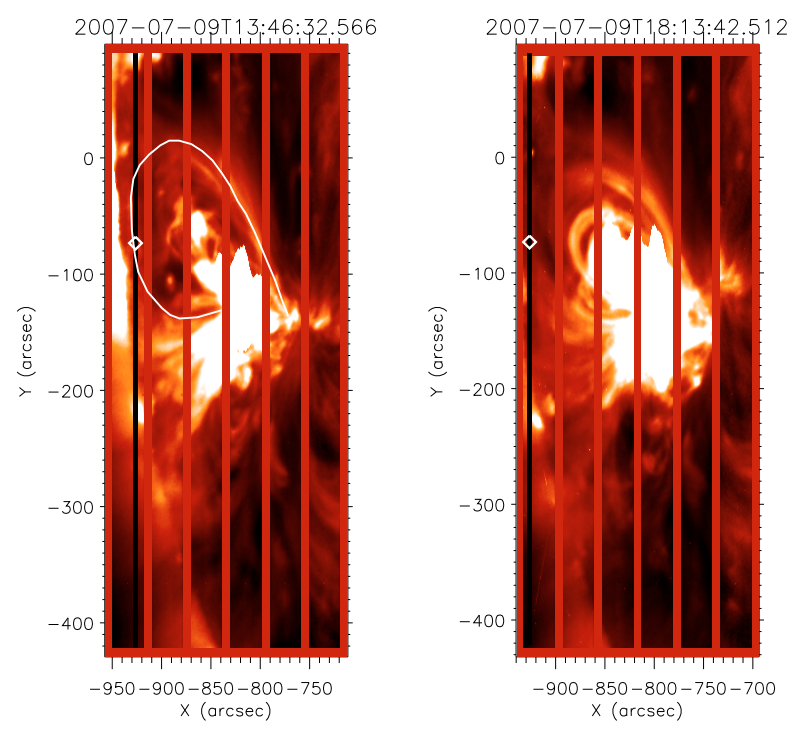

Fig. 1. Overview of the observed active region on 2007 July 9 at 13:46 UT (just after the oscillation) and 18:13 UT, respectively, acquired by Hinode/EIS. The colour code is the intensity in the $195 \AA$ line. The vertical black line indicates the position of the slit. The white diamond indicates the pixel showing oscillations. The white line traces out the oscillating loop.

\section{Observational results}

We analysed data from the EIS instrument (Culhane et al. 2007) onboard Hinode (Kosugi et al. 2007). EIS has both imaging (40" slot) and spectroscopic ( $1^{\prime \prime}$ and $2^{\prime \prime}$ slit) capabilities, in the wavelength range of 170-210 $\AA$ and 250-290 $\AA$. Its spectroscopic mode can operate in a rastering mode (repeated exposures while scanning over the observation target) or a sit-andstare mode (repeated exposures at the same spatial location).

We used sit-and-stare observations of the active region 10963 on 2007 July 9. The observations between 13:18 UT and 13:37 UT are used. Six spectral lines were observed with a cadence time of $12 \mathrm{~s}$ and an exposure time of $10 \mathrm{~s}$ : FeXII 186 and $195 \AA$, FeXIII 196 and $202 \AA$, CaXVII $192 \AA$ and HeII $256 \AA$.

The observations were taken using the "tracking mode" (TR4). Solar rotation can therefore be neglected in the further analysis. An intensity image of the active region taken in the FeXII $195 \AA$ spectral line can be found in Fig. 1. FeXII is most abundant for a temperature of $1.3 \mathrm{MK}$, and the figure can be viewed as a map of the coronal plasma at that temperature. The position of the slit is indicated by a vertical black line.

Hinode is known to have instrumental jitter in its observations. To estimate the jitter in the studied observation, we calculated the correlation between different frames shifted by a number of pixels along the slit and a reference frame. For all frames, the correlation peaked for a shift of 0 pixels. This implied that the jitter in this observation was smaller than 1 pixel and could be neglected.

The raw data was pre-processed by the standard SolarSoft routine eis_prep using the default options (/default,/no_neigh). The spectral lines were fitted by Gaussians using standard routines (eis_auto_fit). The obtained line-of-sight velocities were corrected for the orbital variation of the spacecraft using a sinusoidal fit of the average velocity along the slit (eis_wave_corr). A spline fit of the orbital variation yielded qualitatively identical results.
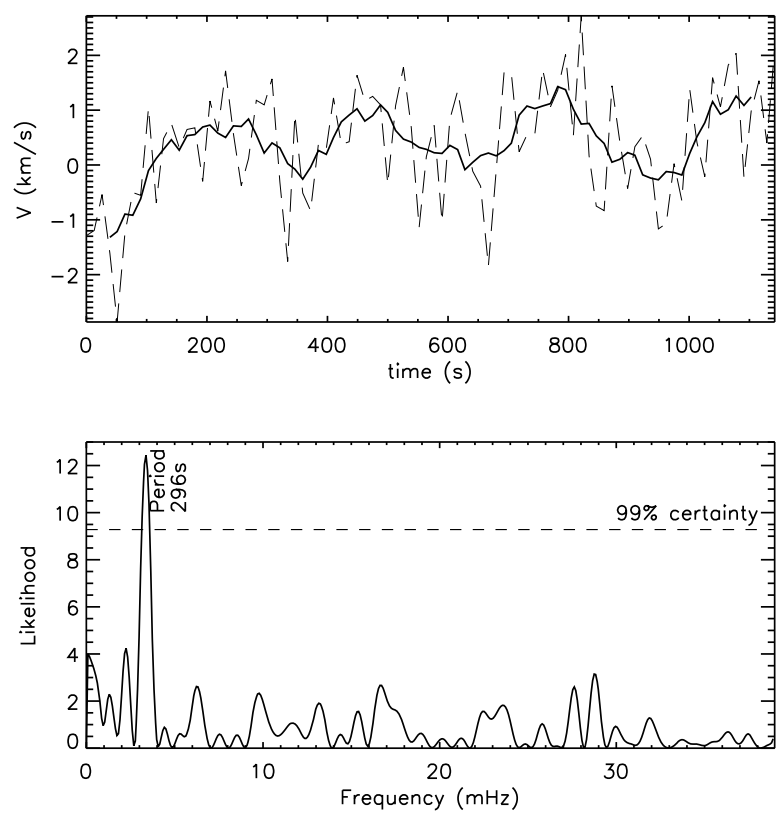

Fig. 2. Top panel: The un-smoothed (dashed line) and smoothed (full line) velocity measurements in the $195 \AA$ line for $y=-72.8^{\prime \prime}$ (indicated with the diamond in Fig. 1). Bottom panel: The Scargle periodogram of the un-smoothed signal.

The un-smoothed line-of-sight velocities measured by the Doppler shift of the $195 \AA$ spectral line at $y=-72.8^{\prime \prime}$ (indicated with the diamond in Fig. 1) are plotted in Fig. 2 (top panel, dashed lines). These un-smoothed velocities were derived using a macro-pixel approach, i.e. average the value in a pixel with its two neighbouring pixels. This reduces the noise level on the data and corresponds to an error in the velocity of $\sim 0.9 \mathrm{~km} \mathrm{~s}^{-1}$.

It is apparent from the time series of the velocity that only three or four periods are present in the current data set. However, this implies that the observation is of relatively high quality, as coronal oscillations are often of an intermittent and temporary nature. Earlier imaging (e.g. Aschwanden et al. 1999; Nakariakov et al. 1999) and spectroscopic observations (e.g. Mariska 2005) have similar limitations.

It is clear from the Scargle periodogram (bottom panel, Fig. 2) that an oscillation is present with a period of $296 \pm 24 \mathrm{~s}$. The full width half maximum of the periodogram is taken as a measure of the error in the period. The presence of the period was established at a confidence level of over $99 \%$. The Scargle periodogram and confidence level are based on the hypothesis that each individual velocity measurement was independent of its subsequent value. The measurement errors in the velocities were taken into account in determining neither the confidence level, nor the estimate of the error in the period. To estimate the importance of the errors in the velocity measurement, we completed Monte-Carlo simulations in which we added Gaussian noise to the original data points. It was found that the error in the period was not influenced by the velocity errors.

When investigating the intensity along the slit (Fig. 3, top panel), it is clear that the oscillations are associated with an intensity enhancement, i.e. a coronal loop. Using the imaging capabilities of EIS, the length of the loop can be measured from the overview images. The loop is observed as a diffuse structure, most clearly visible between $x=\left[-900^{\prime \prime},-800^{\prime \prime}\right]$ and $y=\left[-100^{\prime \prime}, 0\right]$ in the left hand panel of Fig. 1. The loop is traced out by hand and indicated by a white line. The loop is more clearly visible in the right hand panel, which shows an 

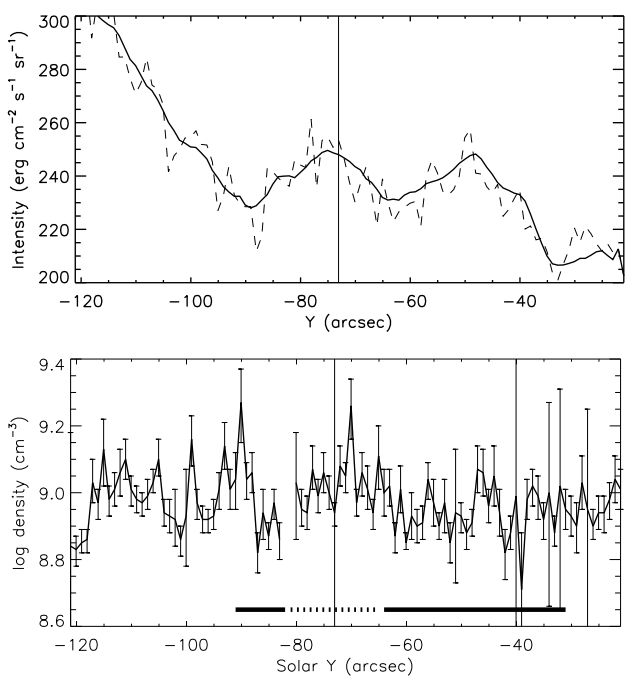

Fig. 3. Top panel: The intensity in the $195 \AA$ line along the slit (--: original data, - : smoothed data). The position with the oscillations (shown with the vertical line) is associated with the density enhancement. Bottom panel: The density derived with CHIANTI using the FeXII 186/195 line ratio. The densities used for the external density are underlined with a full line, whereas the densities used for the internal density are underlined with a dashed line.

image taken $4.5 \mathrm{~h}$ later. From the traced loop, we can estimate the projected loop length and de-rotate it to correct for the effect of the solar longitude, i.e. divide the projected loop length by the cosine of the solar longitude to derive its true length. The loop length was measured to be $390 \mathrm{Mm}$ with an error of approximately $10 \%$, related with projection effects.

Spectroscopic line ratios can be used to derive the density of the emitting coronal plasma (Young et al. 2007), using CHIANTI (Dere et al. 1997; Landi et al. 2006). To use this approach, we first improve the signal-to-noise ratio of the data by averaging the emission over all frames in time. As before, the corresponding intensities are fitted by a Gaussian, taking into account the line blend of the FeXII $186 \AA$ line. The densities are then derived from the ratio of the intensity in the FeXII $186 \AA$ and $195 \AA$ line for each pixel individually. The results of that analysis are displayed in the bottom panel of Fig. 3, which indicates that the density of the oscillating loop is higher than its surroundings at $y=-72.8^{\prime \prime}$ (vertical line). The internal electron density $n_{\text {in }}$ is $9.8 \pm 0.3 \times 10^{14} \mathrm{~m}^{-3}$ and the external electron density $n_{\mathrm{ex}}$ is estimated to be $8.1 \pm 0.2 \times 10^{14} \mathrm{~m}^{-3}$ where we have averaged the density over the pixels underlined by a dotted line and a full line, respectively. It can be seen from the top panel of Fig. 3 that a second loop is present, at the right of the studied loop. Excluding the second loop, an external density within the previous measurement errors is obtained.

Other unrelated positions along the slit also show oscillations at a high confidence level. The currently studied pixel is the most convincing oscillation, and we concentrate on this oscillation alone.

\section{Discussion and coronal seismology}

Before we are able to complete coronal seismology, the oscillation mode must be identified correctly. There are four main MHD modes of solar coronal structures (Nakariakov \& Verwichte 2005): longitudinal (or slow magneto-acoustic), kink (or transverse), sausage, and torsional (or Alfvén).
Alfvén modes assume a torsional character in cylindrical structures, such as loops, which are not necessarily of circular cross-section (Van Doorsselaere et al. 2008). With EIS's spatial resolution, they cannot be observed by Doppler shifts but only by a periodic line broadening. Since the waves are clearly observed by Doppler shifts, this implies that they cannot be interpreted in terms of Alfvén modes.

The sausage mode is a periodic transverse contraction and expansion of the flux tube, which does not produce a net Doppler shift. Furthermore, a sausage mode has strong intensity variations, which is not in the current observation. We are therefore unable to interpret our observation in terms of fast sausage modes.

Slow magneto-acoustic waves have a strong longitudinal velocity component and show intensity variations. For the velocity amplitude that we measure here, the intensity variations would however be below the noise level, which is a few percent of the background intensity. Low harmonic, standing slow magnetoacoustic oscillations can be dismissed easily, because the phase speed $\left(2600 \mathrm{~km} \mathrm{~s}^{-1}\right)$ of the observed wave would be much higher than the local sound speed (a few $100 \mathrm{~km} \mathrm{~s}^{-1}$ ). We cannot exclude completely a running slow magneto-acoustic wave, but there are two important indications that it would be the incorrect interpretation. Firstly, the observed loop is rooted in a sunspot. According to De Moortel et al. (2002), slow magneto-acoustic oscillations in these loops have a shorter period in general than we study here (180 s vs. 300 s). Secondly, running slow waves are excited near the loop foot-points. The detection lengths, over which distance the amplitude decreases by a factor of four, for these oscillations are below $25 \mathrm{Mm}$ (De Moortel et al. 2002). At the height of the current velocity measurement $(\sim 100 \mathrm{Mm})$, this implies that the initial amplitude was reduced by a factor of 250 (when an exponential decay is assumed). In running slow waves, the typical initial density variation is $4 \%$ of the background density (De Moortel et al. 2002). Since in a propagating slow magneto-acoustic wave $\rho^{\prime} / \rho_{0}=V^{\prime} / C_{\mathrm{s}}\left(\rho^{\prime}\right.$ and $V^{\prime}$ are the perturbed density and velocity, $\rho_{0}$ and $C_{\mathrm{s}}$ are the equilibrium density and sound speed), we deduce that the expected value of velocity for a running slow wave is $0.03 \mathrm{~km} \mathrm{~s}^{-1}$ (for a sound speed of $200 \mathrm{~km} \mathrm{~s}^{-1}$ ). The two reasons that we have described provide strong indications that the oscillation that we observe is not a slow magneto-acoustic wave.

The only remaining explanation of the observed oscillations is in terms of fast kink modes. These oscillations explain fully the observed signatures; they are almost incompressible and displace the coronal loop away from its axis. This explanation is also fully consistent with the discovery of low amplitude kink oscillations in the solar corona by the CoMP instrument (Tomczyk et al. 2007; see also Van Doorsselaere et al. 2008), even in the absence of solar flares.

Numerous examples of standing kink waves have been observed (Aschwanden et al. 1999; Nakariakov et al. 1999). The fundamental kink mode was observed to be the most pronounced apart from by the study of De Moortel \& Brady (2007); this and the position at the loop of the pixel with the oscillation, enable the oscillation to be interpreted in terms of the fundamental kink mode.

Using this interpretation, we can deduce a phase speed of $2 L / P=2600 \pm 500 \mathrm{~km} \mathrm{~s}^{-1}$. We can compare this value to the formula for the kink speed $C_{\mathrm{k}}=B / \sqrt{\mu m_{\mathrm{m}} m_{\mathrm{p}}\left(n_{\mathrm{in}}+n_{\mathrm{ex}}\right) / 2}$ (Roberts et al. 1983), where $m_{\mathrm{p}}$ is the proton rest mass and $m_{\mathrm{m}}$ is the mean molecular mass (which we take to be 1.16 for the solar corona). Equating the kink speed to the phase speed and using the previously obtained values for the density, we measure 
the magnitude of the magnetic field in the oscillating loop to be $B=39 \pm 8 \mathrm{G}$. The errors in the current measurement of the magnetic field strength originate mainly in the $10 \%$ errors in both the loop length and the period. The errors in the density measurement do not contribute significantly. The accuracy that we achieve is far superior to the other estimates, such as $13 \pm 9 \mathrm{G}$ in Nakariakov \& Ofman (2001), and represents an improvement on other estimates, for example Ofman \& Wang (2008) who neglected to include the error on the loop length. The relative error can be improved from $70 \%$ and $45 \%$ to $20 \%$.

The modification of the periods of kink modes by the departure from the straight cylindrical model has been considered. In particular, Van Doorsselaere et al. (2004) discovered that for similar transverse structuring the effect of the loop curvature is insignificant $(\$ 6 \%)$. Ruderman (2003) considered a magnetic cylinder of elliptic cross-section, and demonstrated the applicability of the kink mode formalism in that case. Andries et al. (2005b), in addition to McEwan et al. (2006) and Verth et al. (2007), demonstrated that the density stratification effect on the kink mode resonant period is of the order of $10 \%$ and that it controls the ratio of the periods of the global mode and its second harmonics, and potentially the longitudinal structure of the mode. We conclude that the straight cylinder model provides a satisfactory description of the fast kink mode of a coronal loop and can be used to relate the period of the mode to the magnetic field in the loop.

Fast kink oscillations in flaring active regions usually show strong damping. In the current study, however, such a damping effect is not observed. The most probable explanation of the absence of damping is an observational selection effect. If damping was present, the signal would decrease rapidly and blend into the noise; a rapidly damped oscillation could therefore not have been identified in this study. Another option is that the waves we presently observe are similar in nature to the omni-present kink waves observed by Tomczyk et al. (2007). These waves also do not display significant damping, possibly because of the different, non-impulsive excitation of the low-amplitude kink waves as opposed to the flare-induced kink oscillations.

The relation of the damping time to the oscillation period is also observed to differ between coronal structures. For example, kink oscillations studied by Verwichte et al. (2004) were found to decay far less than the oscillations studied by Aschwanden et al. (1999). An adequate theoretical explanation of this effect has not yet been suggested.

\section{Conclusions}

We have reported on the first spectroscopic observation of kink oscillations using EIS onboard Hinode and its application to magnetic field determination. We analysed Doppler oscillations in a coronal loop. The unique simultaneous imaging and spectroscopic capabilities of EIS allow us to measure both the loop length and density, and make EIS excellently suited for coronal seismology. The EIS instrument enables coronal loop oscillations of weak amplitude to be detected that were undetectable with coronal EUV and X-ray imaging instruments. Indeed, if the velocity amplitude is $1 \mathrm{~km} \mathrm{~s}^{-1}$ and the period of oscillations is $300 \mathrm{~s}$, the loop displacement amplitude is about $300 \mathrm{~km}$, which is less than the pixel size of the TRACE EUV imager.

We interpret the oscillation in terms of the fundamental kink mode. Using this interpretation, we have measured for the first time both the loop density and the wave phase speed, inferring a direct measurement of the magnetic field magnitude in the observed coronal loop of $39 \pm 8 \mathrm{G}$. The relative error in this measurement is reduced when compared with earlier measurements. The proof of concept of performing coronal seismology with imaging spectrometers indicates the opportunities EIS offers for the full scale implementation of the MHD coronal seismological technique. Our present analysis could be improved by increasing the exposure times such that a higher signal-tonoise ratio was achieved; the errors in velocity measurements would then be smaller and oscillations would also be observable in other spectral lines. A compromise must however be attained between completing a sufficient number of measurements per period and achieving a high signal-to-noise.

The combination of EIS with the imaging instruments onboard Hinode, which have already demonstrated their potential for the detection of coronal waves and oscillations (e.g. Cirtain et al. 2007; Okamoto et al. 2007; Ofman \& Wang 2008), is also a promising avenue for coronal seismology. We note however that Cirtain et al. (2007); Okamoto et al. (2007) observed propagating kink waves (see Van Doorsselaere et al. 2008, for details) and hence formalism for determining magnetic fields with these waves differs from the method described above. Moreover, the omni-presence of fast magneto-acoustic waves in the corona, discovered by Tomczyk et al. (2007), provides an experimental foundation to the full-scale implementation of coronal seismology.

Acknowledgements. This work was done with support from STFC. The authors would like to thank Dr. J. Noglik for designing the EIS observational campaign.

\section{References}

Andries, J., Arregui, I., \& Goossens, M. 2005a, ApJ, 624, L57

Andries, J., Goossens, M., Hollweg, J. V., Arregui, I., \& Van Doorsselaere, T. 2005b, A\&A, 430, 1109

Arregui, I., Andries, J., Van Doorsselaere, T., Goossens, M., \& Poedts, S. 2007, A\&A, 463, 333

Aschwanden, M. J., Fletcher, L., Schrijver, C. J., \& Alexander, D. 1999, ApJ, 520,880

Cirtain, J. W., Golub, L., Lundquist, L., et al. 2007, Science, 318, 1580

Culhane, J. L., Harra, L. K., James, A. M., et al. 2007, Sol. Phys., 243, 19

De Moortel, I., \& Brady, C. S. 2007, ApJ, 664, 1210

De Moortel, I., Hood, A. W., Ireland, J., \& Walsh, R. W. 2002, Sol. Phys., 209, 89

Dere, K. P., Landi, E., Mason, H. E., Monsignori Fossi, B. C., \& Young, P. R. 1997, A\&AS, 125, 149

Kosugi, T., Matsuzaki, K., Sakao, T., et al. 2007, Sol. Phys., 243, 3

Landi, E., Del Zanna, G., Young, P. R., et al. 2006, ApJS, 162, 261

Mariska, J. T. 2005, ApJ, 620, L67

McEwan, M. P., Donnelly, G. R., Díaz, A. J., \& Roberts, B. 2006, A\&A, 460, 893

Nakariakov, V. M., \& Ofman, L. 2001, A\&A, 372, L53

Nakariakov, V. M., \& Verwichte, E. 2005, Living Rev. Sol. Phys., 2,3

Nakariakov, V. M., Ofman, L., DeLuca, E. E., Roberts, B., \& Davila, J. M. 1999, Science, 285, 862

Ofman, L., \& Aschwanden, M. J. 2002, ApJ, 576, L153

Ofman, L., \& Wang, T. J. 2008, A\&A, 482, L9

Okamoto, T. J., Tsuneta, S., Berger, T. E., et al. 2007, Science, 318, 1577

Roberts, B., Edwin, P. M., \& Benz, A. O. 1983, Nature, 305, 688

Ruderman, M. S. 2003, A\&A, 409, 287

Tomczyk, S., McIntosh, S. W., Keil, S. L., et al. 2007, Science, 317, 1192

Van Doorsselaere, T., Debosscher, A., Andries, J., \& Poedts, S. 2004, A\&A, 424, 1065

Van Doorsselaere, T., Nakariakov, V. M., \& Verwichte, E. 2007, A\&A, 473, 959

Van Doorsselaere, T., Nakariakov, V. M., \& Verwichte, E. 2008, ApJ, 676, L73

Verth, G., Van Doorsselaere, T., Erdélyi, R., \& Goossens, M. 2007, A\&A, 475, 341

Verwichte, E., Nakariakov, V. M., Ofman, L., \& Deluca, E. E. 2004, Sol. Phys., 223, 77

Verwichte, E., Foullon, C., \& Nakariakov, V. M. 2006, A\&A, 452, 615

Young, P. R., Del Zanna, G., Mason, H. E., et al. 2007, PASJ, 59, 857 